\title{
Asynchronous Lectures, View-Speed Effect
}

\author{
Ahmad Mohammadpanah, Nima Atabaki \\ The University of British Columbia, Department of Mechanical Engineering, Canada
}

\begin{abstract}
A study on the effect of speed-watching of recorded lectures in a second, third-, and fourth-year Mechanical Engineering undergraduate courses and one Graduate level course showed that the view speed (i.e. watching lectures with $1.5 \times$ or $2 \times$ times faster than normal speed) has no significant effect on the average grade performance. While the data might suggest that the supposed benefits of being able to speed up a lecture (preserving a lecture content while decreasing the amount of time spending on it) does not have any significant effect on a student's understanding of the Engineering topics content, there might be some disadvantages in speedwatching. Through, a post survey of the same sample groups, majority (82\%) of the speed watcher reported that they feel a bit impatient when they couldn't "speed up" a live conversation. Some reported the feeling of frustration or a lack of attention when they have to attend a live lecture (real-time speed) or inperson lectures.
\end{abstract}

\section{Introduction}

Online and video lectures have become the most common teaching delivery format this year due to the pandemic. With the Online teaching platform, most of university teachers are encouraged/requested to record the live lectures to give students the option to watch the recorded lectures asynchronously. Some teachers may choose to make video lectures outside the regular lecture time and make them available to students to review. The lecture videos like all other common format videos can be viewed with faster speeds (i.e. 1.5, 2, or 2.5 times). The speed-watching piques most of the students' interest, since it preserves a lecture content while decreasing the amount of time spending on it.

The desire to speed watch, recorded lectures may depend on various factors such as personality, English proficiency, or student's workload. It is pretty well established that student learning happens through active engagement with the content rather than passive absorption of lecture materials. It is not clear how much effect does asynchronously watch lectures have. While it is hard to test these factors out, we hypothesize the view speed has minimum effect on student's performance (measured by grades obtained), but it will affect the performance of the students when they switch back to real time speed lectures, i.e., post pandemic.

E-learning and new instructional technologies have been increasingly incorporated into different school learning environment including lecture video recordings as a substitute for live lecture attendance. In the literature, mixed conclusions are presented regarding how this alternative experience impacts students' academic success. In a statistical study by Zureick et al. [1], some of the factors influencing the learning outcome during live and video lectures were examined. At this University, all lectures are videorecorded and videos are made available for streaming and download shortly following the lectures. The Software they use allows students to view, simultaneously and side-by-side, a screen capture of the desktop (usually PowerPoint slides), as well as a video feed. Students have access to the current year's video recordings, but may opt to view the previous year's recordings to stay on pace or ahead of pace with lectures. They studied three cohorts of students of first-year medical students regarding lecture attendance and video usage, focusing on study behaviors that may influence their learning outcomes. Students who reported always attending lectures or viewing lecture videos had higher average histology scores than students who employed an inconsistent strategy (i.e., mixing live attendance and video lectures). Overall, most of the recent studies suggest that both live and video lectures are equally effective. However, we are facing a new challenge caused by high-speed option available with video recorded lectures.

Decline in live lecture attendance has created concern among instructors in various schools to assess students' perceptions, evaluations, and motivations concerning live lectures compared with accelerated, video-recorded lectures viewed online. In this context, a cross-sectional survey study was conducted by Cardall et al. [2] at Harvard Medical School. The survey included all first- and second-year students. The survey questions included topics regarding students lecture attendance; use of class and personal time; use of accelerated, video-recorded lectures; and reasons for viewing video-recorded and live lectures. They were also asked about learning goals satisfaction in live lectures versus video-recorded lectures. The results of this survey reveal that about $30 \%$ of students 
have watched recorded lectures instead of attending live lectures; from which about $88.5 \%$ have used video-accelerating technologies. Despite the fact that live attendance remains the predominant method for viewing lectures, the result of their studies indicated that students find accelerated, video-recorded lectures equally or more valuable. Students' responses to the survey indicate that their decisions to attend lectures or view recorded lectures are motivated primarily by a desire to satisfy their professional goals.

A study by Kristin Wilson et al. [3] showed when students watch a lecture video, mind wandering (MW) increases substantially as a function of time. One solution is to speed lectures up. They tested the impact of speeding up lectures on MW, students' judgment of learning (JOL), comprehension, and self-reported lecture experience (i.e., interest, enjoyment, difficulty, benefit). They reported little negative impact of speeding lectures on comprehension, MW, and JOLs. But, in a different study at University of Kentucky Medical [4] the opposite results were suggested. In order to study the effect of playback at various speeds, two groups of volunteered students were asked to watch two separate videos at $1.5 \mathrm{x}$ speed and the second at $1.0 \mathrm{x}$ speed, then completed assessments following each for the immediate retention of novel material. They concluded, in contrast with the previously published studies that showed improvement in performance with sped-up video-recorded lectures, the performance may be worse. Another study [5] was conducted to scrutinize the effects of playback speed for lecture videos whose layouts consisted of slides together with instructor images. This work, based on comprehension tests and a questionnaire survey, suggests viewing videos at $1.5 \mathrm{x}$ speed is more effective for learning than viewing at normal speed. There are many studies on the videobased education, such as video lecture watching behaviors of learners in online courses [6], Collaborative indexing of lecture video in videobased distance education [7], The instructor as a source of attentional and social cues in video lectures [8], and the effect of instructor's gaze in video lectures [9-10], but there are only limited studies on the effect of speed-watching. These limited studies showed contradictory results and conclusions.

To the knowledge of authors, there is no studies on the effect of speed-watching on Engineering topics, which many of them are required meticulous attention to details and comprehension of formula or laws development. The current study pursues two main objectives. First, to compare the grade performance in exam assessments to determine if playback speed (at $1.5 \mathrm{x}$ or $2 \mathrm{x}$ speed) of recorded lectures has significant different than $1 \mathrm{x}$ speed for the immediate comprehension or retention of topics in Mechanical Engineering courses. Second, to study the potential effect of speed-watching in future learning, i.e. in real life, in-person lectures or discussions when there is not possible to speed up a live conversation.

\section{Methods and Materials}

The University of British Columbia, similar to many other Universities in North America, switched to Online lectures, in 2020 and 2021, due to pandemic. The lectures which have been conducted synchronously, were also recorded, to give students the option for watching them asynchronously for those who could not attend a lecture. The videorecorded lectures have several advantages, include the ability to review, pause and return to finish a lecture later, and watch lectures at faster speeds. This year, at UBC, most of lectures are video-recorded and videos were made available for streaming or sometimes download shortly following the lecture. The Media Gallery playback software (which is available to all students, and is mainly used as a platform to upload the video-recorded lectures) allows students to view, simultaneously a high-quality screen capture of the desktop (usually PowerPoint slides or the Instructor's note), as well as the images or videos capture by Document Camera (typically for showing physical objects - small demos, etc) during a lecture. Typically, most of the instructors keep their webcam On, so students can see the instructor (either side-byside or a small thumbnailed video on the corner of screen). Most of audience (i.e. students, have their webcam off, some opt to turn it on, and it is not mandatory to turn your webcam on). Students have access to the current year's video recordings, and may opt to view the recordings as well as attending the live lectures. Some opt to only watch the recorded lectures, and do not attend the lectures. Attending the live lectures were not mandatory in most of the classes. Those students who do not attend the lectures typically do not attend the tutorial sessions.

An initial anonymous survey of students registered in four Mechanical Engineering undergraduate courses (second year, third, and forth year courses) and one graduate (Master and $\mathrm{PhD}$ students) were conducted to determine the percentage of students who in fact used the feature of fast playback speed.

The assessments in these courses were similar to the regular in-person classes. The grades in these courses were used to evaluate the performance of students who watch with normal speed or watch with faster speeds. We tried to include a variety of courses in this study. The courses were both undergraduate and graduate courses. The students registered in these courses consisted of Mechanical Engineering, Mechatronic Engineering, and Manufacturing Engineering programs. The four undergraduate courses in this study were Machine Dynamics (MANU 265), Manufacturing Processes (MECH 392), Mechanical and Mechatronic Labs and Data Analyses (MECH 305 and 306), and Experimental Thermofluids (MECH 489); and the graduate course 
which was included in this study was Experimental Fluid Mechanics (MECH 582). In this graduate course mainly Master and $\mathrm{PhD}$ students were registered. Some of these courses were offered in Term 1 and some in Term 2 in 2020 and 2021 academic year.

In each of these classes, there were a group of students who attended live online lectures synchronously, and another group who opt to watch the recorded lectures at a later time asynchronously on their own time. With the second group, there were students who watch the videos at normal speed, and a group who choose to watch at higher speeds. It should be emphasized that attending classes were not mandatory and there was no pressure on students to attend the lectures synchronously or to skip the classes and just watch the lectures later. It is also worth mentioning the was not any communication regarding the speed-watching options, i.e. the pros and cons, or any encouragement/discouragement of what students should do. Therefore, we let students to choose what they think is the best for them. We just evaluate their performance, using midterms, assignments, and final grades as the yardstick. In addition, at the end of semester a survey were conducted and asked a few general questions, to see how students self-evaluate the effect of speed watching.

In this study, we treat the group who watched with normal speed same as the group who attended the live lectures. So, we had 5 classes, with each two groups:

Group 1: Watch with normal speed or attend live lectures.

Group 2: Watch the recorded lectures with higher speeds.

For each group we computed the average grades and the standard deviations. An Analysis of Variance (ANOVA), F-test, was also conducted to compare the data and check if there is a significant difference between these two groups in each of these five courses.

\section{Results}

The results of these study showed about 55 presents of students view the recorded lectures with higher speeds (i.e. with $1.5 \mathrm{X}$ or $2 \mathrm{X}$ Times faster), and 45 percent watch the recorded lectures with normal speed or attend the live lectures. The first conclusion was that the speed-watching option was more popular among students. The general average grade performance of the two groups for all the five courses under this study was very close (Only $2 \%$ higher for speed-watcher), with no significant difference in standard deviation of these two groups. A F-test for each of these five courses confirmed that there was no obvious difference in grades between these two groups in each course. Below is a summery of statistical data in each course.

Second year course (MANU 265 - Machine Dynamics), In this undergrad course, the survey showed about $51 \%$ of students view the recorded lectures with higher speeds:

- Third year course (MECH 392 - Manufacturing Processes), In this undergrad course, the survey showed about $68 \%$ of students view the recorded lectures with higher speeds.

- Third year course (MECH 305 and 306 - Data Analyses), In this undergrad course, the survey showed about $57 \%$ of students view the recorded lectures with higher speeds.

- Fourth year course (MECH 489 - Experimental Thermofluids), In this undergrad course, the survey showed about $67 \%$ of students view the recorded lectures with higher speeds.

- Graduate Course (MECH 582 - Experimental Fluid Mechanics), In this graduate course (mainly Master and PhD students), the survey showed about $51 \%$ of students view the recorded lectures with higher speeds.

A detail statistical analyses of one of these undergraduate courses indicates there is not a significant difference between average grades between two groups (normal vs speed watchers). Figure 1 shows the participation percentage in each mode (i.e. synchronous live lectures and then asynchronous with normal or speed watching).

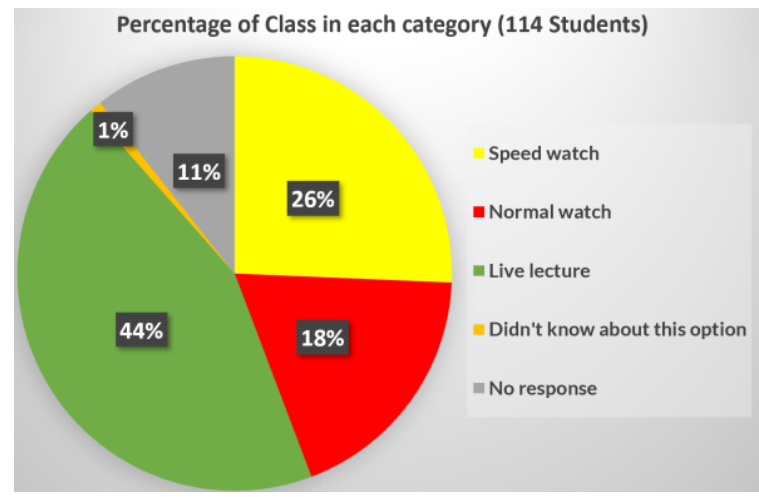

Figure 1. Distribution of Class Attendance

The Speed-Watcher obtained an average grade of 71 with standard deviation of $\mathrm{SD}=16.1$; and the Normal-Watcher average grade was recorded 70 with $\mathrm{SD}=15.7$. As these data indicates, there is not also any noticeable difference between the standard deviations of these two groups. An analysis of Variance (ANOVA) test with confidence level 95\%, 
or significant level $\alpha=5 \%$ confirmed there is not any evidence that these two groups are different.

The grades distribution also indicates a normal distribution of grades. Figure 2 shows the grade distribution.

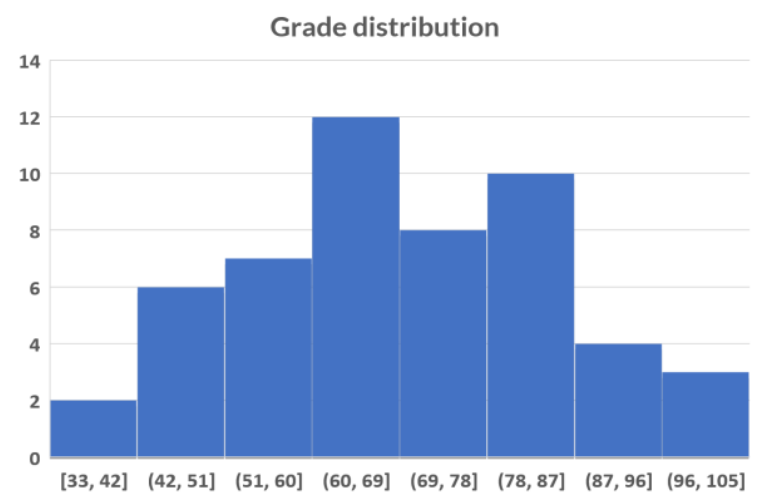

Figure 2. Grade Distribution

Similar statistical analyses in a small graduate level course, with 11 Master and $\mathrm{PhD}$ students shows the average grade of the speed-watcher was only slightly higher (only $1.5 \%$ ) than those who view with normal speed. Later a students' self-evaluation survey on the speed-watching effect, with s single question, "If you are a speed-watcher, in scale of 0 to 100 how effective do you think the speed-watching is for you?" indicated about $76 \%$ of these students believe speedwatching in overall is effective. While this study indicates, over half of the students view the recorded lectures with speeds $1.5 \mathrm{X}$ or $2 \mathrm{X}$, and the average grade performance of the two groups was very close $(1 \%)$, and the sstatistical analysis confirmed there is no obvious deviation in grades between these two groups; the advantages and disadvantages of speed watching is not clear. The authors speculate (based on the survey data) some advantages of speed watching might be saving time and better concentration, with some disadvantages such as feeling impatient when they couldn't "speed up" a live conversation or some reported the feeling of frustration or a lack of attention when they have to attend a live lecture (synchronously).

In addition, an anonymous general survey of several other second, third, and fourth year Mechanical and Manufacturing Engineering undergraduate courses showed, in average over half of students views the lecture with higher speeds. It is well known that sstudent learning happens through active engagement with the content rather than passive absorption of materials. Using the Bloom's taxonomy [11], the speed-watching have smaller effect on student's performance at lower levels learning objectives (e.g. Remembering, Understanding), as shown on Bloom's diagram in Figure 3, but higher effect on higher level (e.g. Analyzing, Creating).

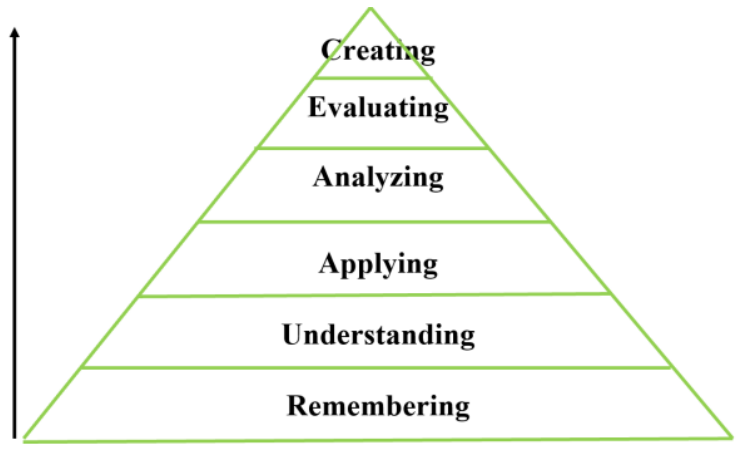

Figure 3. Speed-Watching Effect on each Bloom's Taxonomy Learning Objective

\section{Conclusion}

The data suggested that the supposed benefits of being able to speed up a lecture does not have any significant effect on a student's understanding, at least for these sample size (about 200 students in these courses), there might be some disadvantages in speedwatching. Through, a post survey of the same sample groups, majority (82\%) of the speed watcher reported that they feel a bit impatient when they couldn't "speed up" a live conversation. Some reported the feeling of frustration or a lack of attention when they have to attend a live lecture (real-time speed) or inperson lectures.

\section{References}

[1] Scott Cardall, Edward Krupat, Michael Ulrich (2008). Live lecture versus video-recorded lecture: are students voting with their feet? Acad Med. 83(12):1174-8.

[2] Andrew H Zureick, Jesse Burk-Rafel, Joel A Purkiss, and Michael Hortsch (2018). The interrupted learner: How distractions during live and video lectures influence learning outcomes, Anat Sci Educ 2018 Jul;11(4):366-376.

[3] Wilson, K. E., Martin, L., Smilek, D., and Risko, E. F. (2018). The benefits and costs of speed watching video lectures. Scholarship of Teaching and Learning in Psychology, 4(4), 243-257.

[4] Kristine Song, Amit Chakraborty, Matthew Dawson, Adam Dugan, Brian Adkins, and Christopher Doty, (2018). Does the Podcast Video Playback Speed Affect Comprehension for Novel Curriculum Delivery? A Randomized Trial, West J Emerg Med. 2018 Jan; 19(1): 101-105.

[5] Nagahama, T., Morita, Y., (2017). Effect Analysis of Playback Speed for Lecture Video Including Instructor Images, International Journal for Educational Media and Technology 2017, Vol.11, No. 1, pp.50-58.

[6] Ozan, O., and Ozarslan, Y. (2016). Video lecture watching behaviors of learners in online courses. Educational Media International, 53(1), 27-41. 
[7] Haga, H. (2004). Concept of Video Bookmark (videomark) and Its Application to the Collaborative Indexing of Lecture Video in Video-based Distance Education. International Journal on E-Learning, 3(3), $32-37$.

[8] Stull, A. T., Fiorella, L., and Mayer, R. E. (2020). The case for embodied instruction: The instructor as a source of attentional and social cues in video lectures. Journal of Educational Psychology. Advance online publication.

[9] Pi, Z., Xu, K., Liu, C., and Yang, J. (2020). Instructor presence in video lectures: Eye gaze matters, but not body orientation. Computers and Education, 144, Article 103713.

[10] Wang, H., Pi, Z., and $\mathrm{Hu}$, W. (2019). The instructor's gaze guidance in video lectures improves learning. Journal of Computer Assisted Learning, $35(1), 42-50$.

[11] Bloom, Englehart, Furst, Hill and Krathwohl. (1956). Bloom's Taxonomy of the Cognitive Domain. 